\title{
Autonomous Robot Systems
}

\section{Guest Editorial}

\author{
Luis Almeida · Lino Marques
}

Published online: 2 September 2016

(C) Springer Science+Business Media Dordrecht 2016

This special issue on Autonomous Robot Systems contains updated and extended versions of eight works presented at The $9^{\text {th }}$ IEEE International Conference on Autonomous Robot Systems and Competitions (ICARSC). This conference, scientifically sponsored by the IEEE Robotics and Automation Society (RAS) and by the Portuguese Robotics Society (SPR), takes place every year in a different Portuguese city, as a complementary event to the Portuguese Robotics Open (ROBOTICA), a scientific event composed by robotics competitions and supported by RoboCup. The $9^{\text {th }}$ ICARSC was held in Vila Real, Portugal, on April $8^{\text {th }}-9^{\text {th }}, 2015$.

It was the editors concern to assure two major goals in this edition: firstly to include some of the best works presented at the conference and secondly to provide the reader with a balanced issue, including works addressing the most important topics for robot autonomy. Having these concerns in mind, this edition includes articles spanning from the lower levels of sensing and control, dealing with perception of the

Luis Almeida $(\square)$

Instituto de Telecomunicações, Universidade do Porto,

Porto, Portugal

e-mail: lda@fe.up.pt

\section{Lino Marques}

Instituto de Sistemas e Robótica, Universidade de Coimbra,

Coimbra, Portugal

e-mail: lino@isr.uc.pt operating environment and low-level motion control, passing through the aspects related with the representation of the environment using maps employed to plan paths and support decisions, and finally reaching the higher levels of autonomy, with the integration of learning capabilities into robots able to acquire knowledge and adapt to dynamic environments.

Starting from the lower layer of perception this special issue includes two articles that address issues related to vision. In Robotic Hand Pose Estimation based on Stereo Vision and GPU-enabled Internal Graphical Simulation, the authors Pedro Vicente, Lorenzo Jamone and Alexandre Bernardino propose a novel method that allows estimating the pose of a robot hand while simultaneously calibrating the robot kinematic model in real-time, using a GPU. The method combines stereo vision, proprioception and a 3D computer graphics robot model. In the other article, A Framework for Augmented Reality using Non-Central Catadioptric Cameras, the authors Tiago Dias, Pedro Miraldo and Nuno Gonçalves propose generating information-enhanced images by projecting textured objects onto images acquired with noncentral catadioptric cameras. Their method handles occlusions and illumination/shading areas and it is capable of running up to $20 \mathrm{fps}$ with $1328 \times 1048$ image resolution.

In another layer, concerning motion control, we include two articles particularly applied to humanoid robotics. In Adaptive Robot Biped Locomotion with Dynamic Motion Primitives and Coupled Phase 
Oscillators, the authors José Rosado, Filipe Silva, Vítor Santos and António Amaro present a biped locomotion control framework that learns from human demonstrations combining the modulation of dynamic movement primitives (DMP) with rhythm and phase coordination. This framework also generalizes such demonstrations making them adaptive by adjusting a few control parameters in the learned model, allowing the robot gait pattern to adapt to ground surface irregularities, to step over obstacles and, generally, to tolerate external disturbances. The other article, Contextual Policy Search for Linear and Nonlinear Generalization of a Humanoid Walking Controller, by Abbas Abdolmaleki, Nuno Lau, Luis Paulo Reis, Jan Peters and Gerhard Neumann, presents a flexible robot locomotion controller that copes with multiple contexts. The authors use the recently developed method of contextual relative entropy policy search (REPS) representing contexts with real valued vectors. The article also extends contextual REPS to learn a non-linear policy using radial basis functions (RBF).

Moving up in the logical control architecture, we include three papers that address localization, mapping and planning, the former focusing on multi-robot systems and the latter two focusing on agriculture applications. The first article, MultiRobot Localization and Mapping based on Signed Distance Functions, by Philipp Koch, Stefan May, Michael Schmidpeter, Markus Kühn, Christian Pfitzner, Christian Merkl, Rainer Koch, Martin Fees, Jon Martin, Daniel Ammon and Andreas Nüchter, describes a 2D simultaneous localization and mapping (SLAM) approach that builds a joint map in parallel from 2D LIDAR sensors using signed distance functions (SDF). The resulting framework, implemented with a multi-threaded software architecture, achieves driftreduced pose estimation of the team robots. In Towards a Reliable Robot for Steep Slope Vineyards Monitoring, the authors Filipe Neves dos
Santos, Heber Sobreira, Daniel Campos, Raul Morais, António Paulo Moreira and Olga Contente describe a hybrid SLAM approach named VineSLAM that considers low cost landmarks typically available in vineyards to increase the robot localization accuracy, robustness and redundancy on steep slope irregular terrains. The authors then apply such system to a cost-effective robot for crop monitoring tasks. Conversely, the article Coverage Path Planning for UAVs Photogrammetry with Energy and Resolution Constraints, by Carmelo di Franco and Giorgio Buttazzo, focuses on finding a path that allows an autonomous unmanned aerial vehicle (UAV) to cover a given area of interest for photogrammetric sensing. In particular, the article takes a novel look at coverage path planning (CPP) that jointly considers energy, speed, acceleration, and image resolution. The proposed method checks whether there is energy enough to carry out a desired mission and enforces safe return to launch operation (RTL) operation.

Finally, we include one article addressing knowledgebased high level issues, entitled Experience-based Planning Domains: An Integrated Learning and Deliberation Approach for Intelligent Robots, by Vahid Mokhtari, Luís Seabra Lopes and Armando J. Pinho. It uses experience-based planning domains (EBPD) for task level learning and planning allowing a robot to achieve experience from performing in dynamic environments, to conceptualize each experience with an activity schema and, with these schemata, to make plans for similar situations. The authors illustrate and evaluate their proposed approach in a restaurant environment where a service robot learns complex tasks.

All the submitted papers passed through a rigorous reviewing process being reviewed by at least two specialized reviewers plus the guest editors. The guest editors of this special edition are grateful to the authors of the included papers for their contributions and to the reviewers for their insightful and thorough reviews. 